\title{
Thermophysical effects in laser processing of materials with picosecond and femtosecond pulses
}

\author{
P. P. Pronko, a) S. K. Dutta, and D. Du \\ Center for Ultrafast Optical Science, Department of Electrical Engineering and Computer Science; \\ University of Michigan, Ann Arbor, Michigan 48109 \\ R. K. Singh \\ Department of Materials Science and Engineering, University of Florida, Gainesville, Florida 32611
}

(Received 18 October 1994; accepted for publication 17 July 1995)

\begin{abstract}
Application of picosecond and femtosecond laser pulses to the controlled ablation of materials represents a relatively unexplored yet important topic in laser processing. Such ultrashort pulses are of potential value in areas of thin-film deposition, micromachining, and surgical procedures. We report here some early results of systematic studies being done from the femtosecond to the nanosecond regime, as an assessment of the problems and benefits associated with various laser pulse durations and their use in processing optically absorbing media. Experimental data and theoretical results of computer simulations are presented and compared for the threshold energies of ablation in gold as a function of pulse width from $10 \mathrm{~ns}$ to $100 \mathrm{fs}$. This work is then extended to include further numerically computed results for gold and silicon on ablation rates, threshold surface temperatures, liquid thicknesses, and vaporization rates as a function of pulse duration throughout the ultrafast regime from tens of femtoseconds to a few hundred picoseconds. (1995 American Institute of Physics.
\end{abstract}

\section{INTRODUCTION}

A significant amount of research in pulsed laser ablation and laser processing of materials has been done employing pulses from the nanosecond to the millisecond time regime. These have included wavelengths from the ultraviolet through the visible into the infrared. ${ }^{1}$ Additionally, established work in the area of pulsed laser deposition (PLD) of thin films has been performed largely with excimer lasers operating in the near-UV and with Nd:YAG lasers employing the visible spectrum. ${ }^{2,3}$ More recently, ultrafast pulsed lasers operating throughout the visible and the near-IR have become available and offer a new set of opportunities in the field of laser materials processing with ultrashort pulses that range from picoseconds to femtoseconds. This article explores the physical effects occurring when such fast pulses are used in materials processing for applications in areas such as micromachining, laser ablation, and thin-film deposition. Computer simulation and experimental observations on the vapor phase transformation and other thermal transport effects are examined and related to the thermophysical properties of the materials being studied.

Some earlier work has appeared in the literature concerning the application of short pulses to materials-related issues. Watanabe et al. ${ }^{4}$ and Stern et al. ${ }^{5}$ have explored the use of short pulses for various applications with special attention to surgical procedures. Shank et al. ${ }^{6}$ have investigated the response of semiconductors under femtosecond irradiation in terms of heating, reflectivity, and disorder. Zysset et $a .^{7}$ have investigated materials response using picosecond pulses for optical breakdown in transparent materials, with an interest in applications to ocular tissue and eye surgery. Studies by Du et al. ${ }^{8}$ have more recently extended this earlier work into the femtosecond regime. Issues con-

a)Electronic mail: pronko@eecs.umich.edu cerning the ablation of surface material under short-pulse irradiation have also been examined for polymers, ${ }^{9,10}$ metals, semiconductors, and ceramics. ${ }^{11}$ In particular it has been shown that submicron, subwavelength, and subspot-size holes can be ablated with femtosecond pulses. ${ }^{12}$ Specific studies have also been done on the graphite system regarding its response to laser pulses ranging from nanoseconds to femtoseconds. ${ }^{13-15}$

\section{THERMOPHYSICAL EFFECTS}

In short-pulse materials processing, it is necessary to consider such physical events as internal electron-lattice energy coupling, thermal transport, melting, vaporization, and thermodynamic phase equilibria. All of these primary thermophysical phenomena, at a given power density, will ultimately affect subsequent processes such as threshold for laser ablation, surface temperatures, ablation velocities, plasma excitation, and specific details of atomic or molecular ionization.

Laser pulse absorption and the associated thermal energy transport in materials can be conveniently studied through computer modeling of the heat conduction process with appropriate source and sink terms applicable to the system under investigation. The appearance and evolution of liquid and solid phase boundaries is normally handled by invoking appropriate interfacial boundary conditions that employ a latent heat-dominated moving phase-transition front. ${ }^{16}$ An ablative system can be adequately described in this fashion if account is taken of the partial absorption of the laser pulse by the ablation plasma. ${ }^{17}$ For short pulses it is also necessary to recognize that effects will occur due to the temporal sequence of the appearance and propagation of a finite velocity vapor front. The combined material response will therefore be controlled not only by the physics of coupling the laser energy into the material, but also by the subsequent dynam- 
ics for latent heat transfer in the generation of a liquid-solid interface and a vapor plume. The laser radiation first absorbed by the electrons is subsequently transferred to the bulk crystal atoms over some characteristic time. A description of this process requires a model that considers two different thermal transport media (electrons and ions), which are coupled in some fashion. Mathematically a twotemperature model can be used to describe this system and account for the experimentally observed effects. ${ }^{18-20}$ It is recognized that along with these effects, low- and highdensity, short-scale-length plasmas can be formed. ${ }^{21}$

Heat conduction in laser-irradiated solids is normally treated by considering bulk thermal conductivity as a parameter describing phonon transport and associated thermal energy redistribution. Velocity of sound considerations imply that such an approach is valid in metals for laser pulse times on the order of, or greater than, a few hundred femtoseconds. For example, longitudinal sound wave velocities in gold of $3240 \mathrm{~m} / \mathrm{s}$ result in a sound pulse traveling approximately 15 $\AA$ in $500 \mathrm{fs}$. Additionally, the thermal diffusion distance for a narrow heat pulse is $L=(D t)^{1 / 2}$. A typical thermal diffusivity for metal, such as gold, is $0.4 \mathrm{~cm}^{2} / \mathrm{s}$, where $D=k / C, k$ being the thermal conductivity and $C$ the volume heat capacity. ${ }^{22}$ Based on these considerations a delta-function heat pulse in a metal will spread over approximately $20 \AA$ in a time interval of $100 \mathrm{fs}$. As a consequence, under circumstances where laser-deposited thermal energy is rapidly incorporated into the lattice, the earliest observable initiation of lattice transport of heat can be expected to occur in time intervals of $0.1-1$ ps. The sequence of events leading to observable thermal effects in the lattice is defined more precisely by measurements involving electron-electron thermalization and electron-lattice coupling. Direct measurement of such processes is available from pulse-probe techniques such as those used to monitor time-resolved reflectivity and photoelectron emission. ${ }^{23-26}$ In those works, and more recently as reported by Downer et al. ${ }^{27}$ it is observed that the coupling between energetic electrons and the lattice has a significant temperature dependence and that for high initial electron temperatures $\left(T_{e}>11000 \mathrm{~K}\right.$, as is typical for laserinduced vaporization) one could anticipate very rapid and strong coupling, suggesting that the electrons and lattice are driven toward equipartition of energy within the subpicosecond time domain. In the present work therefore we restrict ourselves, for simplicity, to investigating properties of the dynamic lattice response only. Additional studies are underway investigating the process in terms of a more comprehensive two-temperature coupled model using a temperaturedependent coupling coefficient. That work is the subject of a separate report. ${ }^{28}$ The results presented here are representative of and applicable to very strongly coupled electronlattice systems.

A physical effect that is of particular interest is bulk surface response in the form of a high-velocity vapor interface that moves relative to the underlying material. Both the vapor and the liquid-solid interface will undergo varying degrecs of thermodynamic evolution, with respect to the solid substrate, both during and subsequent to laser pulse absorption. The basic processes occurring in these events are mathematically treatable by classical methods ${ }^{16}$ and have received additional consideration in the case of computer modeling for pulsed laser-solid interactions. ${ }^{17}$ A more detailed discussion of these methods is presented below in Sec. IV.

\section{EXPERIMENT}

Several new laser systems have been under development or are being demonstrated, where high power density $\left(10^{13}-10^{18} \mathrm{~W} / \mathrm{cm}^{2}\right)$, short pulse lengths (picoseconds to femtoseconds), and variable repetition rates are available. ${ }^{29}$ Systems with these characteristics are operating at the Center for Ultrafast Optical Science ${ }^{30}$ at the University of Michigan where one type of design is based primarily on $\mathrm{Ti}^{\mathrm{Al}} \mathrm{Al}_{2} \mathrm{O}_{3}$ lasing media and chirped pulse amplification schemes. ${ }^{31}$ Such a laser $(770 \mathrm{~nm})$ was used in the present experiment where the oscillator is pumped with an argon-ion laser and the amplifier with a doubled Nd:YAG operating at $10 \mathrm{~Hz}$ repetition rate. The stretcher-compressor subsystem is based on grating pairs as the dispersive optical elements. A laser of this type has the capacity to produce a broad range of pulse widths that can be varied over hundrcds of picoseconds. Access to such a broad range of pulses, while not disturbing other important parameters of the experiment such as focusing and beam profile, is an important aspect of this physical setup. In the present experiments the threshold for ablation, defined as the initiation of vaporization, was studied across the pulse range from 150 fs to $7 \mathrm{~ns}$. For pulses up to $1 \mathrm{ps}$ the pulse widths were measured by a single-shot autocorrelator, while those from 10 to 200 ps were measured with a singleshot streak camera. The 7 ns pulses were produced by $Q$ switching the regenerative amplifier without short-pulse injection and measuring the pulse widths using a fast photodiode. A lens with focal length of $25 \mathrm{~cm}$ was used to focus the pulse onto the sample. The focused spot, having a Rayleigh length of approximately $2 \mathrm{~mm}$, was measured in situ with a microscope objective lens and was shown to have a $1 / e$ diameter of $30 \mu \mathrm{m}$. Various metallic sample films were studied to determine their threshold for vaporization followed by laser-induced plasma emission. We report here results of the gold studies. The samples were mounted on a computercontrolled motorized $X-Y$ translation stage. Each location on the sample was illuminated only once by the laser spot.

The threshold for evaporation and plasma breakdown of material was determined by observing the total integrated optical emission from the focal region of the deposited laser pulse. This emission was collected by a lens and directed into a photomultiplier tube through appropriate filters that eliminated the reflected laser light but allowed all other light from optical excitation to be recorded. The data on threshold for breakdown and vaporization is obtained by collecting the emission spectra as a function of pulse energy density based on fluence at the specimen. Since the laser pulse is linearly polarized, the fluence level can be rapidly varied by rotating a polarizer located between two wave plates and simultaneously storing the correlated total emission yield from the photomultiplier in a multichannel analyzer. A schematic diagram of the experimental setup is shown in Fig. 1. The energy of each pulse is measured by the photodiode and stored along with the integrated optical emission from that pulse. 


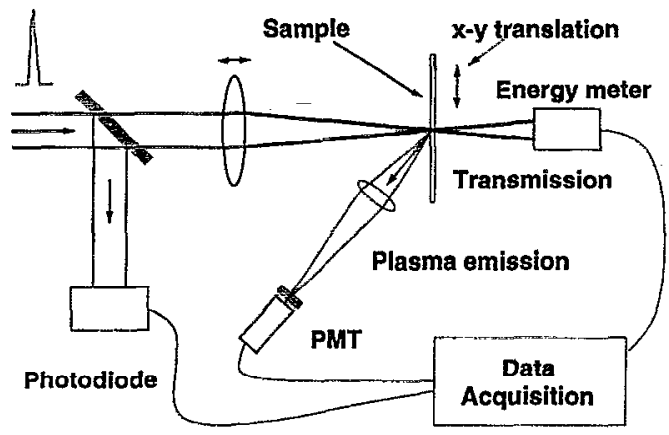

FIG. 1. Schematic of experimental system.

An example of the data obtained from a typical run is presented in Fig. 2. Breakdown threshold is obtained by extrapolating the linear portion of the emission versus fluence yield to the zero emission point as shown in the figure. Data sets like this were produced for various pulse widths to obtain the threshold versus fluence" results presented below. This procedure is expected to yield thresholds close to, or slightly higher than, that for vaporization. Based on consideration of the Saha equation, as applied to singly ionized versus neutral ratios, ${ }^{1,3}$ temperatures slightly above that needed for vaporization will produce measurable amounts of ionization. By extrapolating back to zero emission as shown in Fig. 2, we are assuring that the energy obtained will be closer to the actual threshold for vaporization. Comparison of thresholds obtained in this fashion with those obtained by direct observation using various forms of high-resolution microscopy ${ }^{12}$ and particle emission ${ }^{27}$ confirm the validity of the approach used in this work. Further consideration of these ionization issues is presented at the end of Sec. IV.

\section{COMPUTER MODELING}

In the present work we examine results from solutions of the heat conduction equation using a variable-pulse-length

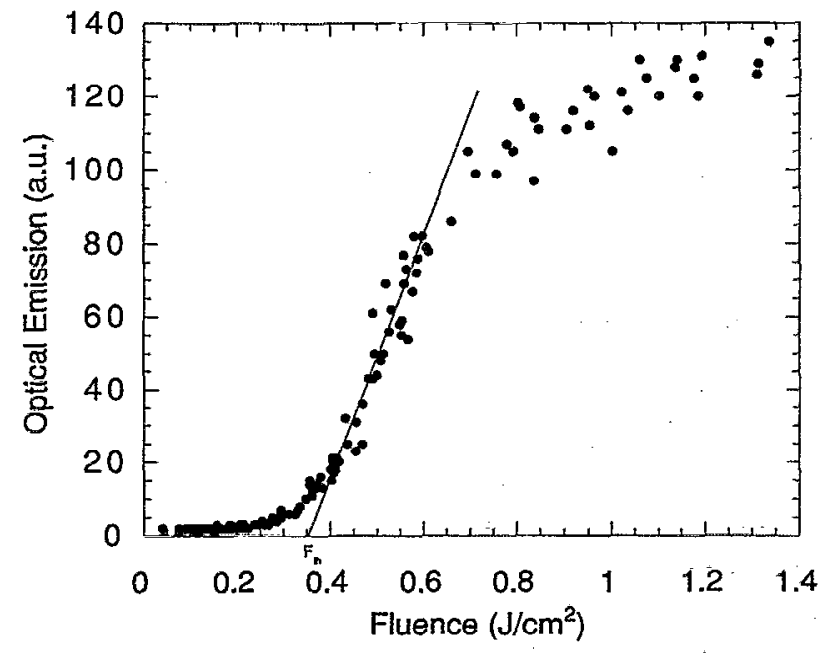

FIG. 2. Integrated optical emission from surface of sample vs laser fluence for $586 \mathrm{fs}$ pulse length. source term to describe the laser energy deposition and to follow the effects that occur when solid, liquid, and vapor phase fronts are present. This model is employed as a tool for evaluating, to first order, how the bulk will respond as a function of variable laser pulse length at threshold and above threshold energies. This approach isolates the effects occurring within the bulk structure of the material. It is instructive to approach the problem this way in order to understand processes associated with the bulk thermophysical response properties of the material as distinct from the more complex electronic response of the system.

Numerical solutions of the heat conduction equation are obtained using the methods of Singh and Narayan. ${ }^{32}$ The temperature at any point in the material during and subsequent to the laser irradiation is given by

$$
\begin{aligned}
\rho_{i}(T) & C_{p_{i}}(T) \frac{\partial T_{i}(x, t)}{\partial t} \\
= & \frac{\partial}{\partial x}\left[K_{i}(T) \frac{\partial T_{i}(x, t)}{\partial x}\right]+I_{0}(t)[1-R(T)] \exp [-\alpha(T) x],
\end{aligned}
$$

where $x$ is the direction perpendicular to the plane of the sample and $t$ is the time from the initiation of the pulse contacting the surface of the material which is at temperature $T$. The equation, through the subscript $i$, describes both the solid and the liquid phases. Temperature-dependent values of density $\rho$, thermal heat capacity $C$, thermal conductivity $k$, reflectivity $R$, and absorption coefficient $\alpha$ may be incorporated into the solutions. A time-dependent incident laser intensity $I_{0}$, is incorporated in Eq. (1) and is used for describing the laser pulse. The solution of the heat conduction equation for the solid and liquid phases is obtained through a dimensionless finite difference technique. ${ }^{32}$ Details of the method are presented in Ref. 32 along with an explanation of how truncation errors are remedied and minimized. In addition, methods for handling the boundary conditions of the liquid-solid and liquid-vapor interface result in important features concerning accessibility of the temperature gradients at these interfaces. These results are subsequently used to calculate the interface velocities.

Melt and vapor phase front propagation is obtained by considering energy heat balance at the vapor-liquid-solid interfaces. The position of the liquid-solid interface is given in terms of the thermal gradients in the solid and liquid. These are defined as follows:

$$
-\left.K_{s} \frac{\partial T_{1}}{\partial x}\right|_{x=s}+\left.K_{l} \frac{\partial T_{2}}{\partial x}\right|_{x=s}=L_{l} \frac{d s}{d t},
$$

where $s$ refers to the position of the interface and $K_{s}$ and $K_{l}$ are the thermal conductivities of the solid and liquid at the interface and $L$ is the latent heat per unit volume of the material. For the case of the vapor front, only one thermal gradient is present in the equation, that being in the liquid. For that case $L$ will be the latent heat of vaporization and $d s / d t$ will be the velocity of the vapor front. The position of the vapor front at any time $t$ after absorption of the laser pulse will then be given in terms of 


$$
\left.K_{l} \frac{\partial T}{\partial x}\right|_{s}=L_{v} \frac{d s}{d t} . \quad \text { or } \quad S=\left.\int_{0}^{t^{\prime}} \frac{K_{l}}{L_{v}} \frac{\partial T}{\partial x}\right|_{s} ^{s} d t
$$

The appearance of a vapor species will therefore be generally given as the time $t^{\prime}$ required for $s$ to be some significant fraction of an atomic layer, this being controlled by the ratio of the thermal conductivity to the latent heat for vaporization. Since this ratio is always finite, it is expected that the appearance of the vapor species will always occur with some finite speed. The relationship between the time for vapor formation and the length of the laser pulse is obviously of significance in terms of how the material responds as a function of the laser pulse duration. The problem is essentially one of defining a surface through which the latent heat of vaporization (or melting) must be transported in order for the front to continue moving into (or out of) the material. The rate at which such transport can occur will be controlled both by the lattice thermal conductivity and the local thermal gradient. The finite velocity of the vapor interface $V_{s}$, is therefore given as

$$
V_{s}=\left(\frac{k_{l}}{L_{v}}\right)\left(\frac{d T}{d x}\right)_{s},
$$

where $L_{v}$ is the latent heat of vaporization and the thermal gradient is evaluated at the vapor-liquid interface. A consequence of this finite interface velocity is the introduction of a certain time delay into the thermal relaxation of the system. This time retardation of the vapor front movement, relative to the energy deposition of the pulse, results in a competitive inward bulk diffusion of heat energy relative to its loss through the surface as a result of vaporization. This effect has consequences on such properties as energy threshold for material removal under fast-pulse irradiation as well as the maximum surface temperature achieved as a function of pulse length for energies above threshold. Demonstration of these effects are presented below for the case of gold and silicon.

An additional feature included in the present model is a simplified absorption parameter that accounts for partial absorption of the laser pulse by the plasma as it develops out of the vaporization process. ${ }^{17}$ As discussed in Sec. III on threshold measurements, a distinction can be drawn between the onset of vaporization and the appearance of a luminous plasma. The two phenomena are related through vapor pressure as a function of temperature and the ratio of thermally ionized species compared to neutrals. In local thermodynamic equilibrium the Saha equation typically predicts $0.01 \%$ ionization at the onset of vaporization (i.e., $2873 \mathrm{~K}$ in gold), with a very rapidly increasing ionized fraction developing as the temperature proceeds upward from that point. This will happen as the laser pulse continues to be absorbed by both the vapor plasma and the solid. At about $5500 \mathrm{~K} \mathrm{a}$ $1 \%$ ionization could be expected with $10 \%$ at $7500 \mathrm{~K}$ and $80 \%$ at $10000 \mathrm{~K}$. Eventually a fully ionized plasma will develop. Continued heating of the bulk solid will depend on the fraction of the pulse absorbed by the plasma and the fraction reaching the surface of the material. Nonlinear absorption in the plasma by inverse bremsstrahlung increases

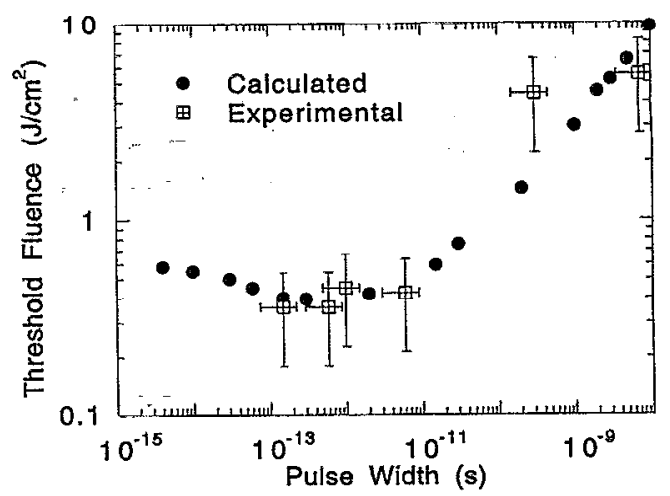

FIG. 3. Threshold fluence vs pulse width for gold at $770 \mathrm{~nm}$ (experimental and computed modeling results).

rapidly, proceeding as the square of the electron density and the cube of the charge state resulting in a partitioning of laser energy between the plasma and the bulk material. In order to account for this in the present model, the vaporization process at the surface of the bulk is shielded by an expanding plasma according to the following equation:

$$
P_{p}=P_{\text {ind }}\left[1-\exp \left(-a_{p} d_{p}\right)\right] \text {, }
$$

where $P_{p}$ is the power absorbed by the plasma and $P_{\text {inc }}$ is the instantaneous total power density incident from the laser. The absorption coefficient is $a_{p}$ and $d_{p}$ is the scale length of the plasma normal to the surface. Our purpose here is to look at the plasma's relative effect on the vaporization process as the laser pulse length decreases from the nanosecond to the femtosecond time scale. The role of the plasma absorption term will vary depending on the origination time of the plasma relative to the temporal length of the laser pulse. Details of the absolute values of the plasma absorption coefficient and its variation with plasma parameters, though important, are of lesser concern in the present work compared to the relative effects the plasma produces as a consequence of decreasing pulse duration. A simplified procedure for making this evaluation involves establishing a relationship such that

$$
a_{p} d_{p}=\alpha_{\mathrm{sim}} \Delta x_{v} .
$$

Here the product of the true plasma absorption coefficient and the plasma scale length are replaced with a simulated product consisting of an effective average absorption coefficient $\alpha_{\text {sim }}$ multiplying the vapor thickness $\Delta x_{v}$ as given by the computer model. ${ }^{17,32}$

\section{PRESENTATION OF RESULTS}

\section{A. Gold-770 nm}

Total integrated optical emission yields, as shown in Fig. 2 , were produced from a gold surface for pulse widths ranging from 150 fs to $7 \mathrm{~ns}$ using laser radiation at $770 \mathrm{~nm}$. Experimental thresholds, as a function of pulse width, were determined by the extrapolation technique of Sec. III and the results presented in Fig. 3. Also presented in Fig. 3 are the numerical computer results for the vaporization thresholds. The physical parameters used in the simulation model are 
TABLE I. Physical parameters for computer simulation using square temporal pulse shape.

\begin{tabular}{|c|c|c|}
\hline & Gold & Silicon \\
\hline Wavelength (nm) & 770 & 308 \\
\hline Melting temp. (K) & 1336 & 1685 \\
\hline Vaporization temp. (K) & 2873 & 3540 \\
\hline \multicolumn{3}{|l|}{ Latent heat $\left(\mathrm{J} \mathrm{cm}^{-3}\right)$ : } \\
\hline Liquid & 1245 & 4206 \\
\hline Vapor & 30489 & 32020 \\
\hline $\begin{array}{l}\text { Thermal } \\
\text { conductivity: } \\
\left(\mathrm{W} \mathrm{cm} \mathrm{cm}^{-1} \mathrm{~K}^{-1}\right)\end{array}$ & $\begin{array}{c}T<1336 \mathrm{~K}:-6.87 \\
\times 10^{-4} T+3.4 \\
T>1336 \mathrm{~K}: 1.16\end{array}$ & $\begin{array}{ll}T<1370 \mathrm{~K}: & 1585 \times T^{-1.23} \\
T>1370 \mathrm{~K}: & 0.221 \\
T>1685 \mathrm{~K}: & 0.700\end{array}$ \\
\hline $\begin{array}{l}\text { Volume heat } \\
\text { capacity: } \\
\qquad\left(\mathrm{J} \mathrm{cm} \mathrm{cm}^{-3} \mathrm{~K}^{-1}\right)\end{array}$ & $7.25 \times 10^{-4} T+2.3$ & $\begin{array}{l}2.0+2.54 \times 10^{-4} T^{-1} \\
-3.68 \times 10^{4} T^{-2} \\
T>1685 \mathrm{~K}: 2.54\end{array}$ \\
\hline \multicolumn{3}{|l|}{ Reflectivity: } \\
\hline Solid & 0.94 & 0.59 \\
\hline Liquid & 0.94 & 0.73 \\
\hline \multicolumn{3}{|l|}{ Absorption coeff. $\left(\mathrm{cm}^{-1}\right)$ : } \\
\hline Solld & $7.27 \times 10^{5}$ & $8 \times 10^{5}$ \\
\hline Liquid & $7.27 \times 10^{5}$ & $8 \times 10^{5}$ \\
\hline Vapor & $5.0 \times 10^{5}$ & $5 \times 10^{5}$ \\
\hline
\end{tabular}

given in Table I as obtained from standard references. ${ }^{22}$ The experimental and computed ablation threshold values are compared and brought into coincidence in the 1 ps regime through adjustment of the optical reflectivity in the theoretical calculation. The extrapolation technique produces a threshold that is defined as the fluence needed to bring the system up to, but not actually at, the onset of optical emission from a radiating plasma or electronically excited vapor as observed with a photomultiplier tube (PMT). For the computer simulations, the ablation threshold is defined as the fluence needed to initiate the loss of material as a consequence of removing a few angstroms of the surface during vaporization. As physical entities, these two thresholds can be slightly different and therefore require a way to be calibrated for comparison. They are normalized to each other by choosing a value of optical pulse reflectivity that brings a selected portion of the computed threshold data into coincidence with the measured values. In the present case this matching is done in the pulse width range of $1 \mathrm{ps}$ and is accomplished by using a laser pulse reflectivity of $94 \%$ (i.e., $6 \%$ of the pulse is absorbed). This reflectivity is comparable to that reported in the literature by others. ${ }^{18-20,22}$ Comparison of the modeling and experimental results demonstrates reasonable agreement, considering the uncertainties in the data, and therefore supports the approach taken in the modeling calculation for pulse widths down to $150 \mathrm{fs}$. For pulse lengths shorter than this, one could expect to see consequences of delayed electron-lattice coupling. Efforts to obtain experimental data for shorter pulses are currently underway, as well as comparisons to an improved computer model that explicitly considers such coupling effects.

It is seen in Fig. 3 that for pulses longer than 100 ps the threshold varies as the square root of the pulse width. This is a direct consequence of the threshold for ablation being con- trolled primarily by the thermal diffusion of the input pulse energy. The longer the pulse length the more time is available for the heat to diffuse into the bulk before the vapor stage is achieved. As the pulses go below 100 ps a gradual but definite departure from the $t^{1 / 2}$ behavior begins. Throughout the $1 \mathrm{ps}$ regime an approximately constant threshold is in effect. This may be considered a consequence of the thermal diffusion length $(D t)^{1 / 2}$ being on the same scale of, or shorter than, the bulk laser absorption depth $(1 / \alpha)$. Inward bulk thermal diffusion does not become appreciable until after the pulse is over. For the case of $770 \mathrm{~nm}$ radiation in gold this depth, as determined from the bulk metal optical extinction coefficient, ${ }^{21}$ is approximately $15 \mathrm{~nm}$. The dominant characteristics associated with the response of the material for pulse widths below this range will be determined by other factors.

The process becomes more complex with further reduction in pulse width for both the computed and experimental data. Other thermophysical effects are occurring throughout this range that have an effect on the threshold curve. These are

(1) the finite velocity of the vapor front relative to the underlying material and

(2) the structure of the thermal gradients that are at the surface where the vapor is supported and deeper within the material where the bulk diffusion occurs.

The minimization and subsequent increase in threshold with decreasing pulse width, as seen in the modeling results, is a manifestation of these phenomena. As the pulse time decreases, for any given fluence, the thermal gradients at the surface and in the bulk will increase. The finite velocity of the vapor front will determine the relaxation of the nearsurface gradient while bulk diffusion will determine the temperature profile deeper in the material. These two gradients together control the net transport of energy out of the irradiated zone and will collectively determine, at any given pulse duration, the relative amount of energy lost to the vaporization of material and the relative amount being lost to the bulk (see Fig. 9). As more of the energy is drawn into the bulk an increase in the threshold for vaporization will result. The roles of these thermal gradients are magnified as a result of their extreme steepness, being in the range of $25 \mathrm{~K} / \AA$ as determined from the computer results. Gradients of this magnitude will, however, still result in subsonic thermal and vapor transport.

It should also be recognized that the incoming pulse is interacting with solid, liquid, and vapor as it goes through the entire process of being absorbed at or near the surface of the material. The computer simulations take these processes into account by including their associated effects in the calculations. For the longer pulse lengths a significant portion of the pulse is absorbed in the liquid as well as in the plasma. As the pulse length decreases a commensurate change occurs in the fraction of the pulse that is distributed among these differing phase states. An example of this is shown in Fig. 4 where a plot is presented of the fraction of a $0.6 \mathrm{~J} / \mathrm{cm}^{2}$ pulse that is subject to absorption as a consequence of exposure to the vapor plasma. It is seen that in the $10 \mathrm{ps}$ range about $20 \%$ 


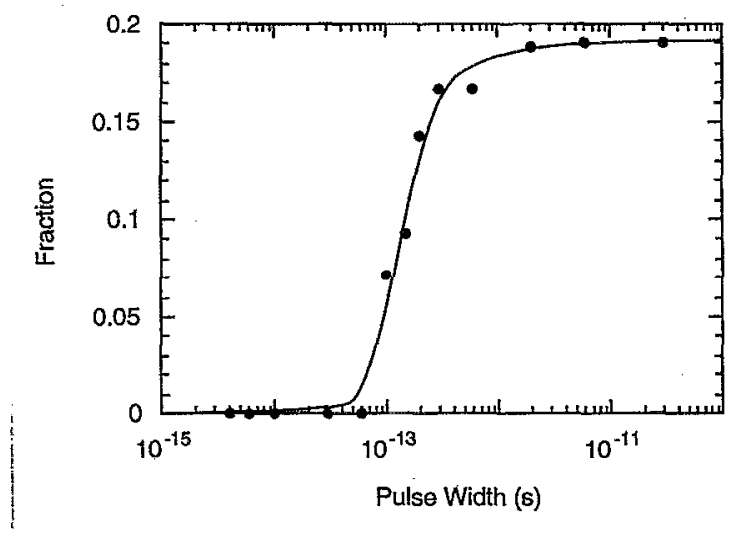

FIG. 4. Fraction of pulse subject to absorption as result of exposure to vapor as a function of pulse width for gold at $770 \mathrm{~nm}$.

of the pulse is exposed to the vapor, whereas a rapid drop in this fraction occurs below about 1 ps and it becomes negligibly small below 100 fs. Thus, for these shorter pulses, energy that might otherwise be lost to the plasma is being absorbed and stored by the material in a superheated (liquid) state at the surface of the film. The consequence of this is an increase in the maximum surface temperature, at the threshold for ablation, as is shown in Fig. 5. Such elevated threshold surface temperatures will obviously have consequences for those situations where thin-film deposition with short pulses is of interest.

\section{B. Silicon-308 nm}

Computed results for $308 \mathrm{~nm}$ pulses in silicon are presented in Figs. 6-8, below. We have no experimental data available for comparison at this wavelength. The parameters used in the simulation model are presented in Table I. Thresholds for vaporization in silicon are shown in Fig. 6. As in the case of gold, the threshold fluences are significantly dependent on pulse width for pulses above and below the 1-10 ps range. For pulses above this range a thermaldiffusion-dominated process is in evidence, whereas below this range other thermophysical processes become important.

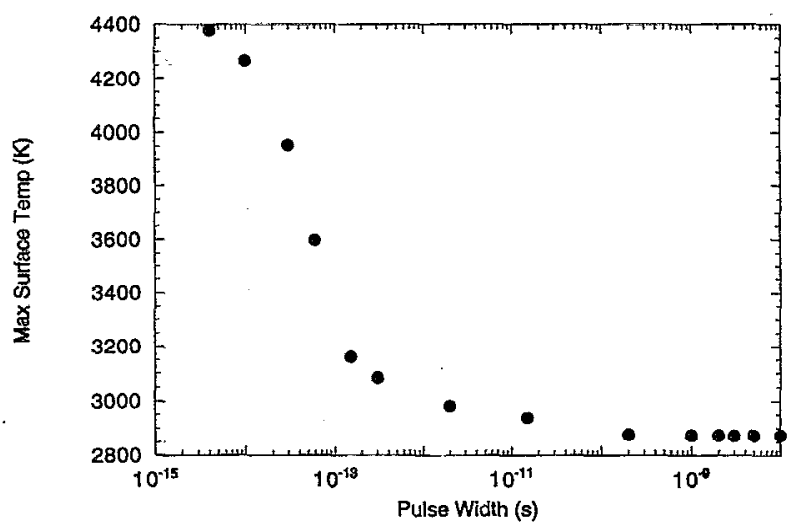

FIG. 5. Maximum surface temperature at threshold vs pulse width for gold at $770 \mathrm{~nm}$.

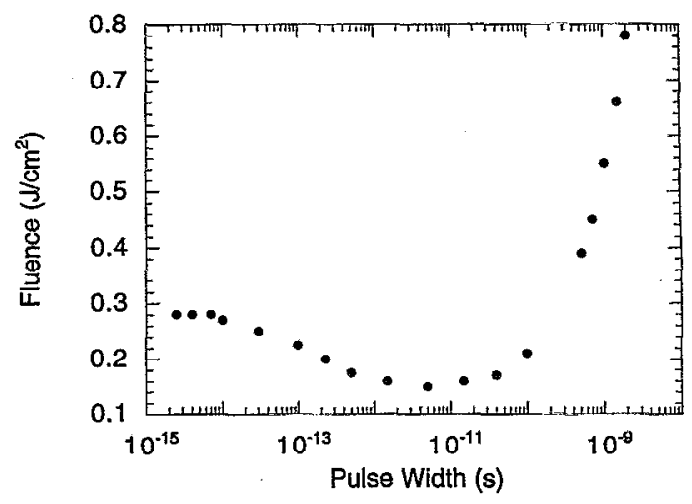

FIG. 6. Threshold fluence for silicon at $308 \mathrm{~nm}$ vs pulse width.

As with gold (Fig. 5), the maximum surface temperature at threshold is found to dramatically increase for pulses below $1 \mathrm{ps}$. The shape of this threshold curve, as a function of pulse width, is characteristically similar to that of gold and is explained by the same processes. Details vary to some extent, however the basic structure is the same with similar physical effects being responsible in both the gold and silicon cases.

In the present modeling situation it is possible to follow the vapor thickness removed and the thickness of the liquid formed as a function of pulse duration. Shown in Fig. 7 are the amount of liquid and vapor formed in silicon, where the laser fluence of $0.35 \mathrm{~J} / \mathrm{cm}^{2}$ is well above threshold for vapor ablation as defined by Fig. 6. The liquid thickness at first increases with decreasing pulse width due to reduction in diffusional loss of heat, but then reverses this trend once a vapor state begins to form. Since vaporization represents a cooling process at the surface, it causes the liquid thickness to reverse its trend at the onset of vaporization. Additionally, some of the incoming pulse can be absorbed in the vapor at these ps pulse durations. The liquid thickness continues decreasing with decreasing pulse width due to a finite liquidsolid interface velocity that minimizes the thickness while a steepening thermal gradient draws heat into the bulk. By way of compensation, the simulations show that a superheating of the liquid occurs as the pulse width decreases, since insuffi-

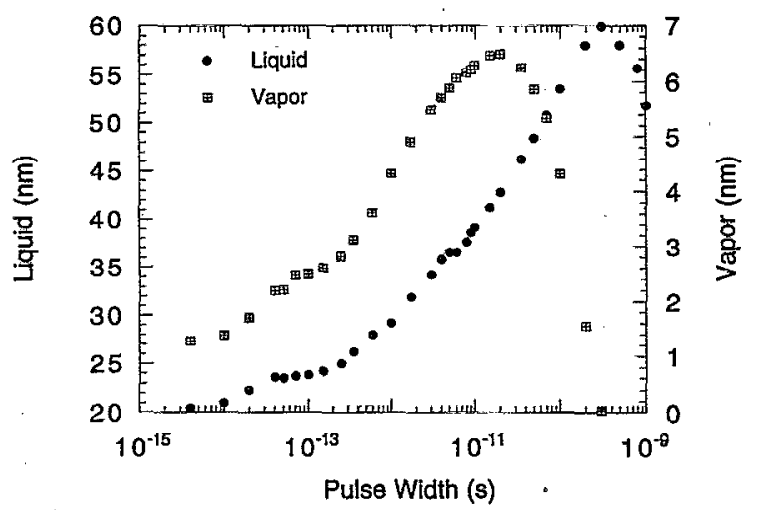

FIG. 7. Vapor and liquid thickness vs pulse width for silicon above threshold $\left(0.35 \mathrm{~J} / \mathrm{cm}^{2}\right)$ at $308 \mathrm{~nm}$. 


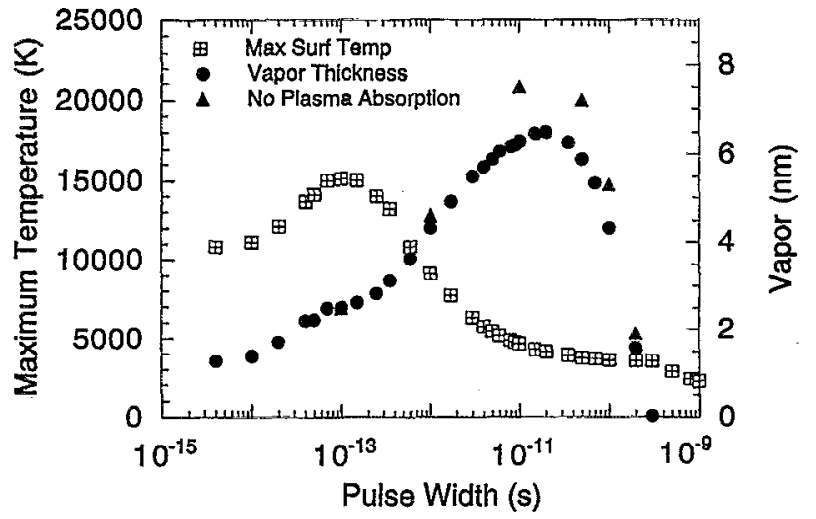

FIG. 8. Maximum surface temperature and vapor thickness vs pulse width in silicon for $0.35 \mathrm{~J} / \mathrm{cm}^{2}$ at $308 \mathrm{~nm}$. Shown also is vapor thickness in the absence of plasma absorption.

cient thermal loss occurs while the phase front velocity remains finite. In terms of the vapor thickness removed, it is seen to first increase with decreasing pulse duration, to reach a maximum at about $20 \mathrm{ps,} \mathrm{and} \mathrm{then} \mathrm{rapidly} \mathrm{to} \mathrm{decrease} \mathrm{as}$ the pulse duration is decreased further. These results are once again associated with the change in response of the system above and below about $10 \mathrm{ps}$, where, for longer pulse times, thermal diffusion and plasma absorption dominate, whereas for shorter times other thermophysical phenomena, such as finite vapor front velocity and competing thermal gradients, become controlling as explained above for the case of gold. Since the results in Fig. 7 are for an incident energy above threshold, the vapor will provide an absorbing medium for part of the incoming pulse. The effect of having absorption in the vapor compared to not having it present are seen in Fig. 8. Generally speaking, the amount of absorption in the vapor increases with the amount of vapor present as expressed in Eq. (5). It will be noticed that as the pulse widths approach the $100 \mathrm{fs}$ mark the effects of the absorbing vapor disappear. This is a consequence of the fact that the time required to launch the vapor is longer than the pulse time, and so no absorption of the pulse occurs in the vapor. In further consideration of the liquid part of the system, it is clear that energy which is used to form the vapor is otherwise unavailable for production of liquid. This is manifested as a decreasing liquid thickness with decreasing pulse duration once the vapor starts to form (see Fig. 7). It is observed that the liquid dynamics are much simpler than that of the vapor in that the liquid is capable of undergoing brief superheating as a way of compensating for the finite velocity of the vapor front and the minimization of inward thermal diffusion. Equivalent attempts by the vapor to superheat are short circuited by its uniform conversion to an absorbing plasma. No provisions for a time-dependent plasma formation are incorporated into the model since it is assumed to be uniformly heated by the incoming pulse. Increases in maximum surface temperature, as seen in Figs. 5 and 8, are manifestations of the liquid undergoing superheating since it is at the surface of the material throughout the period and directly exposed to a maximum of laser absorption. Physically this liquid superheating could lead to much higher vapor temperatures when the delayed vapor does begin to evolve suggesting that very hot plasmas can be formed from this effect.

\section{DISCUSSION AND CONCLUSION}

Using computer modeling and experimental data on thresholds, we have studied the thermophysical response of absorbing material as a function of fluence and pulse time across the nanosecond to femtosecond time scale. Several

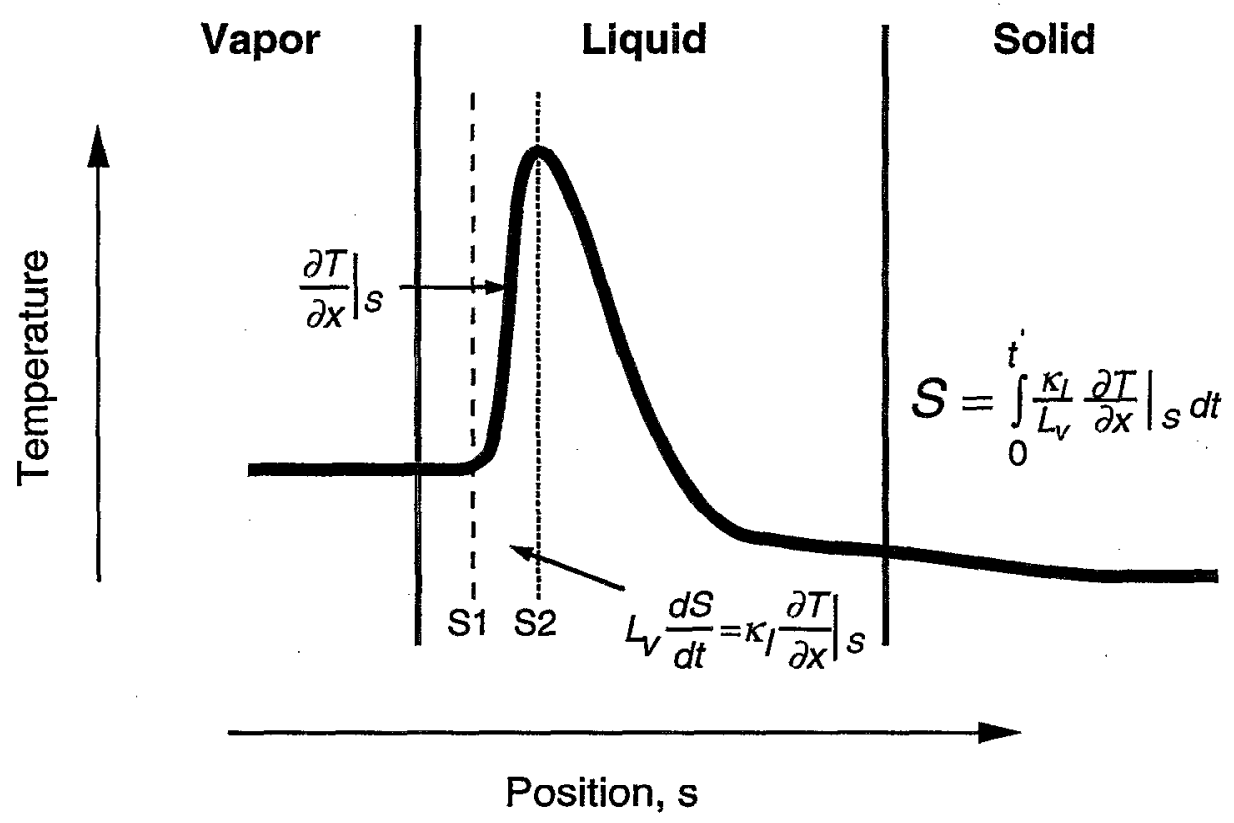

FIG. 9. Schematic diagram of bulk thermal gradients and effect on thermal diffusion vs vaporization. 
interesting new effects have been identified and examined regarding thermophysical phenomena that have varying consequence and that occur over a range of pulse durations. As the laser pulse time decreases below approximately $1 \mathrm{ps}$ it is recognized that electron-lattice coupling properties could become a factor. Since there is evidence that the electronlattice system is strongly coupled at elevated temperatures in the bulk, we have focused our attention on the contributions to the materials response arising from lattice thermal diffusion, associated thermal gradients, and phase-state interface velocity effects. These are common thermophysical effects for all matcrials and as such mcrit detailed consideration. It is observed, in the results of our experiments and computer simulations, that bulk thermal diffusion of the incident laser energy has consequences on the threshold for ablation, as well as for the thickness of liquefied and vaporized material. As the pulse lengths become shorter and enter the picosecond and subpicosecond regime, it is observed that the ablation threshold and vapor thickness are more dominantly controlled by the thermal gradient that is formed at the vaporsurface interface along with its relationship to the thermal gradient that extends into the bulk of the material. Figure 9 shows a schematic drawing of the temperature profiles in this near-surface region. It is seen that as a consequence of the vaporization cooling at the surface, a steep positive-slope thermal gradient will be present. Farther into the bulk a second negative-sloped gradient exists resulting from, and driving, the inward diffusion of heat to the bulk. Since both the vaporization and bulk diffusion processes are thermal gradient driven, as described in Eqs. (1) and (4), the two opposite gradients compete with each other for the energy budget of the absorbed laser pulse. The side with the steeper gradient will tend to dominate the energy extraction process. Thus, depending on pulse times and internal temperature profiles, one can observe decreasing or increasing vaporization thresholds and vaporization thicknesses. Surface temperatures are observed to be dominated by pulse width effects for pulse times that are less than vaporization initiation times. In some cases an incoming pulse may be exposed to and interact with a significant fraction of the vapor, whereas in other cases the pulse may be short enough to see no appreciable vapor at all. For extremely short pulses the appearance of the vapor could be sufficiently delayed and decoupled from the initial pulse so that steep thermal-gradient-driven diffusion remains the only initially significant effect. For longer pulses the normal latent heat and conventional vaporization process will control surface temperatures. All of the effects discussed above will have, in one way or another, consequences in the use of ultrafast pulses for materials processing.

\section{ACKNOWLEDGMENTS}

$\mathrm{X}$. B. Liu provided assistance with experimental data acquisition, J. Squier contributed valuable help in laser setup, and $\mathbf{J}$. Viatella helped in resolving computer code problems. This work was supported in part by the National Science Foundation through the Center for Ultrafast Optical Science under STC PHY 8920108.
'J. F. Ready, Effects of High-Power Laser Radiation (Academic, New York, 1971).

${ }^{2}$ Laser Ablation in Materials Processing: Fundamentals and Applications. edited by B. Braren, J. J. Dubowski, and D. P. Norton, Symposium Proceedings of the Materials Research Society, Vol. 285 (MRS, Pittsburgh, 1992).

${ }^{3}$ Pulsed Laser Deposition of Thin Films, edited by D. B. Chrisey and G. K. Hubler (Wiley, New York, 1994).

${ }^{4}$ S. Watanabe, R. R. Anderson, S. Brorson, G. Dalickas, J. G. Fujimoto, and T. J. Flotte, Photochem. Photobiol. 53, 757 (1991).

${ }^{5}$ D. Stern, R. W. Schoenlein, C. A. Puliafito, E. T. Dobi, R. Birngruber, and J. G. Fujimoto, Arch. Ophthalmol. 107, 587 (1989).

${ }^{6}$ C. V. Shank, R. Yen, and C. Hirlimann, Phys. Rev. Lett. 50, 454 (1983); Phys. Rev. Lett. 51, 900 (1983); C. V. Shank and M. C. Downer, Mater. Res. Soc. Symp. Proc. 51, 15 (1985).

${ }^{7}$ B. Zysset, J. G. Fujimoto, and T. F. Deutsch, Appl. Phys. B 48, 139 (1989); B. Zysset, J. G. Fujimoto, C. A. Puliafito, R. Bingruber, and T. F. Deutsch, Lasers in Surgery and Medicine 9, 193 (1989).

${ }^{8}$ D. Du, X. Liu, G. Korn, J. Squier, and G. Mourou, Appl. Phys. Lett. 64, 3071 (1994).

${ }^{9}$ S. Kuper and M. Stuke, Appl. Phys. B 44, 199 (1987).

${ }^{10}$ S. Prcuss, M. Spath, Y. Zhang, and M. Stuke, Appl. Phys. Lett. 62, 3049 (1993).

${ }^{11}$ W. Kautek and J. Kruger, in Laser Materials Processing: Industrial and Microelectronics Applications, edited by E. Beyer, M. Cantello, A. V. La Rossa, L. D. Laude, F. O. Olsen, and G. Sepold, Proc. SPIE Vol. 2207 (SPIE, Bellingham, WA, 1994), p. 600.

${ }^{\text {I2} P . ~ P . ~ P r o n k o, ~ S . ~ K . ~ D u t t a, ~ J . ~ S q u i e r, ~ J . ~ V . ~ R u d d, ~ a n d ~ G . ~ M o u r o u, ~ O p t . ~}$ Commun. 114, 106 (1995).

${ }^{13}$ A. M. Malvezzi, N. Blocmbergen, and C. Y. Huang, Phys. Rev. Lett. 57, 146 (1986).

${ }^{14}$ D. H. Reitze, X. Wang, H. Ahn, and M. C. Downer, Phys. Rev. B 45, 2677 (1992).

${ }^{15}$ F. Muller, K. Mann, P. Simon, J. S. Bernstein, and G. J. Zaal, Proc. SPIE 1858, 464 (1993)

${ }^{16} \mathrm{H}$. S. Carslaw and J. C. Jaeger, Conduction of Heat in Solids, 2nd ed. (Oxford University Press, Oxford, 1959).

${ }^{17}$ R. K. Singh and J. Narayan, Phys. Rev. B 4, 8843 (1990); J. Appl. Phys. 68, 233 (1990).

${ }^{18}$ S. I. Anisimov, B. L. Kapeliovich, and T. L. Perelman, Zh. Eksp. Teor. Fiz. 66, 776 (1974).

${ }^{19}$ C. K. Sun, F. Vallee, L. Acioli, E. P. Ippen, and J. G. Fujimoto, Phys. Rev. B 48, 12 (1993).

${ }^{20}$ H. E. Elsayed-Alyi, T. B. Norris, M. A. Pessot, and G. A. Mourou, Phys. Rev. Lett. 58, 1212 (1987).

${ }^{21}$ M. M. Murnane, H. C. Kapteyn, M. D. Rosen, and R. W. Falcone, Science 251, 531 (1991).

${ }^{22}$ American Institute of Physics Handbook, 2nd ed. (McGraw-Hill, New York, 1963); CRC Handbook of Chemistry and Physics, edited by R. C. West and M. J. Astle (CRC, Boca Raton, FL, 1979).

${ }^{23}$ G. L. Eesley, Phys. Rev. B 33, 2144 (1986).

${ }^{24}$ R. W. Schoeniein, W. Z. Lin, J. G. Fujimoto, and G. L. Eesley, Phys. Rev. Lett. 58, 1680 (1987); S. D. Brorson, J. G. Fujimoto, and E. P. Ippen, ibid. 59, 1962 (1987).

${ }^{25}$ W. S. Fann, R. Storz, H. W. K. Tom, and J. Bokor, Phys. Rev. Lett. 68 , 2834 (1992).

${ }^{26}$ H. E. Elsayed-Ali, T. Juhasz, G. O. Smith, and W. E. Bron, Phys. Rev. B 43, 4488 (1991).

${ }^{27}$ M. C. Downer, R. L. Fork, and C. V. Shank, J. Opt. Soc. Am. B 2, 595 (1985); X. Y. Wang, D. M. Riffe, Y.-S. Lee, and M. C. Downer, Phys. Rev. B 50, 8016 (1994).

${ }^{28}$ P. P. Pronko and R. K. Singh (nnpublisher).

${ }^{29}$ M. C. Richardson, SPIE Solid State Lasers II 1410, 15 (1991).

${ }^{30} \mathrm{~J}$. Squier and G. Mourou, Laser Focus World, June 1992; J. Squier, F. Salin, G. Mourou, and D. Harter, Opt. Lett. 16, 325 (1991); F. Salin, J. Squier, G. Mourou, and G. Vaillancourt, Opt. Lett. 16, 1965 (1991).

${ }^{31}$ D. Strickland and G. Mourou, Opt. Commun. 56, 219 (1985).

${ }^{32}$ R. K. Singh and J. Narayan, Mater. Sci. Eng. B 3, 217 (1989). 